\title{
Recent progress on mechanisms of human cognition and brain disorders
}

\author{
Fang Fang ${ }^{1,2,3^{*}} \&$ Hailan $\mathrm{Hu}^{4,5,6^{*}}$ \\ ${ }^{1}$ School of Psychological and Cognitive Sciences and Beijing Key Laboratory of Behavior and Mental Health, \\ Peking University, Beijing 100871, China; \\ ${ }^{2}$ IDG/McGovern Institute for Brain Research, Peking University, Beijing 100871, China; \\ ${ }^{3}$ Peking-Tsinghua Center for Life Sciences, Peking University, Beijing 100871, China; \\ ${ }^{4}$ Department of Psychiatry of First Affiliated Hospital, Zhejiang University School of Medicine, Hangzhou 310003, China; \\ ${ }^{5}$ The MOE Frontier Science Center for Brain Research and Brain-Machine Integration, School of Brain Science and Brain Medicine Zhejiang \\ University, Hangzhou 310012, China; \\ ${ }^{6}$ NHC and CAMS Key Laboratory of Medical Neurobiology, Mental Health Center, Zhejiang University, Hangzhou 310058, China;
}

Received April 26, 2021; published online May 10, 2021

Citation: Fang, F., and Hu, H. (2021). Recent progress on mechanisms of human cognition and brain disorders. Sci China Life Sci 64, 843-846. https://doi.org/ $10.1007 / \mathrm{s} 11427-021-1938-8$

Human cognition plays a crucial role in our daily life. It enables us to perceive a constant influx of inputs, to memorize the scenarios, and to reason or decide their outcomes (Thagard, 2005). Abnormal cognitive function, which is usually involved in neurological and mental diseases, such as chronic pain, mood disorders, autism, dementia, often seriously deteriorates individuals' life quality and brings inexhaustible social problems. According to the China Mental Health Survey (CMHS) data in 2019, nearly 200 million people in China are suffering from mental illness, which puts heavy financial pressure on society and affects social stability (Huang et al., 2019). Facing this challenge, early screening, diagnosis, and intervention of brain disorders based on the principles of cognitive neuroscience are in urgent need.

The relationship between human cognition and brain disorders is closely intertwined. On one hand, brain disorders help to advance our understanding of human cognition. For instance, inspired by the evidence from patients with brain impairments, Paul Broca linked word production to the "Broca's area" (Paul, 1861). On the other hand, the devel-

*Corresponding authors (Fang Fang, email: ffang@pku.edu.cn; Hailan Hu, email: huhailan@zju.edu.cn) opment of cognitive neuroscience, especially the novel brain imaging techniques, has brought a lot of new insights to subserve our understanding of the neural mechanisms underpinning brain disorders (Purves et al., 2008). In this special issue, we review the latest research progress on cognition from the aspects of vision, audition, pain, and decision making, as well as on brain disorders including mood disorders, autism, and Alzheimer's disease (AD). We hope that this special issue will stimulate more interest in related topics and bring idea exchanges between cognitive neuroscience and mechanistic studies of brain disorders.

Vision plays a central role in human cognition. In visual cognition, there is a clear dissociation between the rich visual experience and the limited amount of information available to report. This dissociation has led to an old philosophical and scientific debate: is visual consciousness confined by reportability? The overflow hypothesis suggests our visual consciousness is far beyond, not limited by, what we can report. In the review, Hui Chen and colleagues first systematically reviewed existing evidence that supports the overflow argument, as well as the opposing ones (Fu et al., 2021). They then introduced a series of their recent findings based on a striking phenomenon called attribute amnesia to 
support the overflow hypothesis. This review provides novel evidence and insights on an old debate.

Auditory cognition is a key area in human cognition. Deficits in the auditory system may lead to severe brain disorders, such as autism. Autism patients, featured with deficits in social communication and repetitive activities, have atypical cognitive phenotypes. The review paper given by Suiping Wang and colleague (Yu and Wang, 2021) discussed recent researches focusing on early auditory development in autism. Normal audition is one of the basic requirements of speech learning and language acquisition. From this standpoint, the authors discussed whether the previous studies that focused on the role of speech and language development in autism spectrum disorders did not sufficiently consider the developmental abnormalities in basic auditory functions. They first introduced the auditory development stages in typically developing children, and then systematically summarized the evidence of abnormal auditory development in autism. Furthermore, the authors examined the links between abnormal audition and speech development by discussing the disadvantageous phonetic processing and auditory scene analysis in autism. In all, this informative review pointed out that future researches may reconsider the contributions of sensory and higher-order dysfunctions in autism.

Pain is an intensive cognitive experience. Chronic pain is the persistent pain after healing of damage or disease. It has become one of the most common reasons for physician visits (Hart et al., 1995). The experience of chronic pain does not arise from persisting activation of peripheral nociceptors, but from pathological changes in brain circuits. To uncover the brain dysfunctions underlying chronic pain, advanced brain imaging and data analytical approaches have been applied. $\mathrm{Li}$ $\mathrm{Hu}$ and his colleagues reviewed clinical discoveries of chronic pain and pain interventions with magnetic resonance imaging (MRI) technique, as well as emerging studies combining machine learning and neuroimaging (Tu et al., 2020). The authors first introduced the working principles of MRI and conventional data processing approaches. Then they listed clinical MRI data of abnormal neuronal activities in different brain regions from patients suffering multitype chronic pain. Next, they reviewed brain responses of clinical pain interventions such as pharmacotherapy, neuromodulation, and acupuncture. Furthermore, they presented the latest biomarker analysis with the combination of brain imaging and machine learning. Finally, the authors concluded that future studies should target and modulate these biomarkers to study the causal relationships between brain and chronic pain, moving from neural observations towards mechanistic manipulations.

Decision-making is a cognitive process that requires the use of multiple cognitive functions. In daily life, people make social decisions that are influenced by both self and other-regarding preferences (Fehr and Camerer, 2007). In their review paper, Jian Li and Yinmin Ni (Ni and Li, 2020) reviewed recent advances in the cognitive and neural mechanisms underpinning the social learning and decisionmaking. The authors first summarized how people infer others' internal state during social learning and how social factors such as social comparison, fairness, trust and reciprocity, guilt and gratitude, and social norm compliance affect social decision-making. They proposed that social learning and decision-making could be considered as the extension of the individual learning and decision-making framework. Together, this informative review highlighted the future research directions on how to interpret the social information in the complex social environment. Meanwhile, the review gave insights into how to coordinate different brain regions to shape social interactions.

Emotions have not been regarded as a cognitive process traditionally, but accumulating researches on animal models have aroused interest in studying the cognitive psychology of emotions in humans (Phelps, 2006). Mood disorders, including major depressive disorder (MDD) and bipolar disorder (BD), have become the most common mental disorders affecting hundreds of millions of people worldwide. Yiru Fang and his colleagues reviewed multiple standard tests to measure affective cognition abnormalities, as well as clinical results of abnormal affective cognition in patients of mood disorders (Chen et al., 2021). The patients exhibit significant deficits in cognitive evaluations. They also have trouble correctly interpreting emotional faces, words, speeches, pictures, etc. Also, the patients have difficulties in regulating their mood after negative experience, or making appropriate decisions during reward and punishment tasks. These results suggested that future depression therapies should target not only the affective cognitive dysfunctions, but also the functions of emotion interpretation, emotion regulation and reward-based decision making.

Great progress has been made in past decades to reveal the pathophysiological mechanism of mood disorders, especially MDD. Most theories focus on abnormalities of neurotransmitters and neuronal circuits. As research evidence begins to accumulate, microglia, the resident immune cells inside the brain, are discovered to play an important role in depression. The review paper presented by Hailan $\mathrm{Hu}$ and her coworkers discussed the latest perspectives on the role of microglia in MDD (Jia et al., 2020). The authors firstly reviewed dynamic microglial properties of both morphology and molecular profiles in response to depression-related stress. They then summarized possible mechanisms of how microglia contribute to depression. In response to abnormal neuronal activities, signal molecules or hormones under stress condition, microglia secret cytokines and other metabolites, which cause neuroinflammation and modulate depression-related neural circuits. Finally, the review discussed the effects of antidepressants on microglia. Both traditional 
antidepressants and novel drugs like ketamine affect microglial states and may exert their antidepressant effects through microglial mechanisms. Thus, understanding the role of microglia in depression should facilitate the development of new therapeutic strategies to benefit patients with this severe mental disorder.

$\mathrm{AD}$ is another major brain disorder that leads to cognitive and affective deficits. AD has become the most common type of dementias and a major challenge to our aging society. A hallmark of $\mathrm{AD}$ is senile plaques composed of amyloid- $\beta$ $(A \beta)$. Accordingly, $A \beta$ has been suggested to play a pivotal or causative role in the pathogenesis of $\mathrm{AD}$, therefore, is a main target of drug development. Unfortunately, all clinical trials that target $A \beta$ have failed to improve the cognition of patients in phase 3 clinical trials so far. In this issue, Yanjiang Wang and colleagues re-evaluate whether $A \beta$ is a valid therapeutic target of $\mathrm{AD}$, discussing critical concerns of the current anti-A $\beta$ therapeutic strategies (Sun et al., 2020). In addition to $A \beta$, simultaneous targeting to the multiple pathologies of $\mathrm{AD}$ pathogenesis, tertiary prevention strategies, and multidomain intervention targeting risk factors and comorbidities are also proposed to improve the therapeutic efficiency of AD treatments.

Tremendous progress has been made on cognitive neuroscience and its clinical applications related to brain diseases, thanks to the rapid development of modern research techniques including new anatomical methods, tools for recording and manipulating neuron activity, multi-omics analysis and advanced data processing technology. With the support of the China Brain Project (Poo et al., 2016), we believe that high-quality researches in the current fields in China will bloom soon. To address urgent societal needs on brain health, future researches should not only focus on discovering principles of cognition or mechanisms of brain disorders alone, but endeavor to bridge these two fields for the development of new therapeutic strategies.
Compliance and ethics The author(s) declare that they have no conflict of interest.

\section{References}

Chen, J., Wang, Z., Li, Z., Peng, D., and Fang, Y. (2021). Disturbances of affective cognition in mood disorders. Sci China Life Sci doi: 10.1007/ s11427-020-1898-2.

Fehr, E., and Camerer, C.F. (2007). Social neuroeconomics: the neural circuitry of social preferences. Trends Cogn Sci 11, 419-427.

Fu, Y., Yan, W., Shen, M., and Chen, H. (2021). Does consciousness overflow cognitive access? Novel insights from the new phenomenon of attribute amnesia. Sci China Life Sci doi: 10.1007/s11427-020-1831-8.

Hart, L.G., Deyo, R.A., and Cherkin, D.C. (1995). Physician office visits for low back pain: Frequency, clinical evaluation, and treatment patterns from a U.S. national survey. Spine 20, 11-19.

Huang, Y., Wang, Y., Wang, H., Liu, Z., Yu, X., Yan, J., Yu, Y., Kou, C., $\mathrm{Xu}, \mathrm{X} ., \mathrm{Lu}$, J., et al. (2019). Prevalence of mental disorders in China: a cross-sectional epidemiological study. Lancet Psychiatry 6, 211-224.

Jia, X., Gao, Z., and Hu, H. (2020). Microglia in depression: current perspectives. Sci China Life Sci doi: 10.1007/s11427-020-1815-6.

$\mathrm{Ni}$, Y., and Li, J. (2020). Neural mechanisms of social learning and decision-making. Sci China Life Sci doi: 10.1007/s11427-020-1833-8.

Paul, B. (1861). Remarks on the seat of the faculty of articulated language, following an observation of aphemia. Bull Mem Soc Anat Paris 36, 330-357.

Phelps, E.A. (2006). Emotion and cognition: insights from studies of the human amygdala. Annu Rev Psychol 57, 27-53.

Poo, M.M., Du, J.L., Ip, N.Y., Xiong, Z.Q., Xu, B., and Tan, T. (2016). China Brain Project: basic neuroscience, brain diseases, and braininspired computing. Neuron 92, 591-596.

Purves, D., Cabeza, R., Huettel, S.A., LaBar, K.S., Platt, M.L., Woldorff, M.G., and Brannon, E.M. (2008). Cognitive Neuroscience. Sunderland: Sinauer Associates, Inc..

Sun, B.L., Chen, Y., Fan, D.Y., Zhu, C., Zeng, F., and Wang, Y.J. (2020). Critical thinking on amyloid-beta-targeted therapy: challenges and perspectives. Sci China Life Sci doi: 10.1007/s11427-020-1810-y.

Thagard, P. (2005). Mind: An Introduction to Cognitive Science. Cambridge: The MIT Press.

Tu, Y., Cao, J., Bi, Y., and Hu, L. (2020) Magnetic resonance imaging for chronic pain: diagnosis, manipulation, and biomarkers. Sci China Life Sci doi: $10.1007 / \mathrm{s} 11427-020-1822-4$.

Yu, L., and Wang, S. (2021). Aberrant auditory system and its developmental implications for autism. Sci China Life Sci doi: 10.1007/s11427-020-1863-6. 


\section{Biographical Sketch}
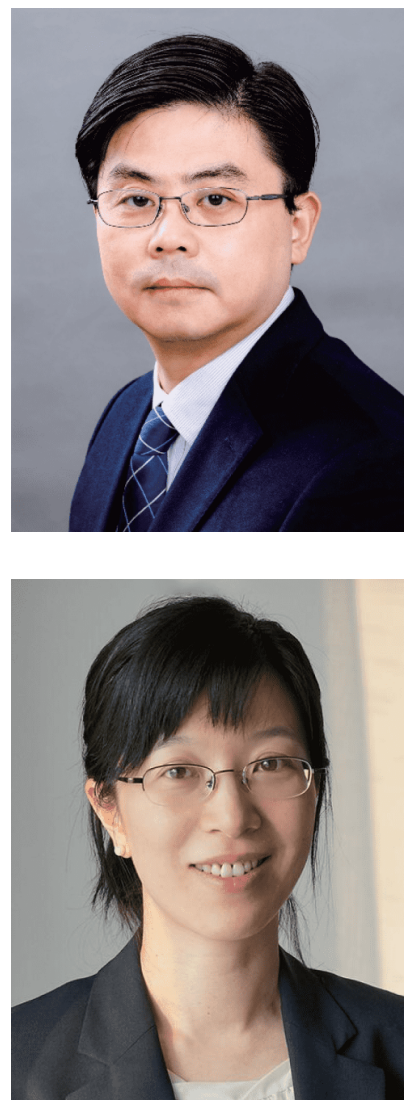

Dr. Fang Fang is Professor of Psychology, Dean of the School of Psychological and Cognitive Sciences, and Executive Associate Director of the IDG/McGovern Institute for Brain Research at Peking University. He obtained a Ph.D. in Cognitive and Biological Psychology at the University of Minnesota in 2006, and was a Postdoctoral Research Associate between 2006 and 2007. His research seeks to understand the neural mechanisms of visual and cognitive processes by combining neuroimaging, brain stimulation, psychophysics, computational modeling, and human genetics. Topics under investigation include visual learning and adaptation, visual attention and awareness, object and face perception. He received the Young Investigaor Award: Basic Science from the International Union of Psychological Science (IUPsyS) in 2016 and was elected as a Fellow of the Association for Psychological Science (APS) in 2018. He currently serves on the editorial board for Current Biology, Experimental Brain Research, and Science China Life Sciences.

Hailan Hu is Director of the MOE Frontier Center of Brain and Brain-machine integration at Zhejiang University. She holds a BA from Beijing University and a PhD from UC Berkeley, and was previously on the faculty at the Institute of Neuroscience, Chinese Academy of Sciences. Her laboratory seeks to understand how emotional and social behaviors are encoded and regulated in the brain, with a main focus on the neural circuitry underlying depression and social dominance. Her team has demonstrated that social hierarchy ranking is positively correlated with and causatively regulated by synaptic strength of neurons in the dorsal medial prefrontal cortex. Based on this, she further identified the neural mechanism underlying the winner effect, by which individuals increase their chance of winning after previous victories. Her recent work has uncovered a new model to explain the etiology of depression and the rapid antidepressant actions of ketamine, involving NMDA receptordependent burst activity of lateral habenular neurons. Her work has led to the identification of several molecular targets for developing new antidepressant drugs. Dr. Hu is a recipient of the National Outstanding Youth Award, Tan Jiazhen Life Science Innovation Award, Top 10 Science Progress Award of China, Ho Leung Ho Lee Foundation Prize and IBRO-Kemali international Prize. 